\title{
Surface Effects on the Vibration and Buckling of Double-Nanobeam-Systems
}

\author{
Dong-Hui Wang and Gang-Feng Wang \\ Department of Engineering Mechanics, SVL, Xi'an Jiaotong University, Xi'an 710049, China \\ Correspondence should be addressed to Gang-Feng Wang, wanggf@mail.xjtu.edu.cn
}

Received 13 June 2011; Accepted 18 August 2011

Academic Editor: Raymond Whitby

Copyright () 2011 D.-H. Wang and G.-F. Wang. This is an open access article distributed under the Creative Commons Attribution License, which permits unrestricted use, distribution, and reproduction in any medium, provided the original work is properly cited.

\begin{abstract}
Surface effects on the transverse vibration and axial buckling of double-nanobeam-system (DNBS) are examined based on a refined Euler-Bernoulli beam model. For three typical deformation modes of DNBS, we derive the natural frequency and critical axial load accounting for both surface elasticity and residual surface tension, respectively. It is found that surface effects get quite important when the cross-sectional size of beams shrinks to nanometers. No matter for vibration or axial buckling, surface effects are just the same in three deformation modes and usually enhance the natural frequency and critical load. However, the interaction between beams is clearly distinct in different deformation modes. This study might be helpful for the design of nano-optomechanical systems and nanoelectromechanical systems.
\end{abstract}

\section{Introduction}

Nanowires hold a wide variety of potential applications, such as sensors, actuators, transistors, probes, and resonators in nanoelectromechnical systems (NEMSs) [1]. In the design of nanowire-based components, it is of great importance to acquire the mechanical behaviors of nanowires accurately. Owing to the increasing ratio of surface area to volume in nanostructured components, the influence of surfaces gets important in their mechanical performance. To account for surface energy in solids, Gurtin et al. [2] established the surface elasticity theory, and recently its applications in nanosized structural element agree reasonably well with atomistic simulations and experimental measurements [36]. For example, Miller and Shenoy [3] investigated the size-dependent elastic properties of nanoscale beams and plates by both surface elasticity and atomic simulation. Ru [4] explained the difference and essence of various versions of Gurtin's surface elastic theory. A core-shell model was developed by Chen et al. [5] to explain the size-dependent Young's modulus of $\mathrm{ZnO}$ nanowires. Through LaplaceYoung equation, Wang and Feng $[7,8]$ addressed both the impacts of residual surface stress and surface elasticity on the vibration and buckling of nanobeams. He and Lilley
[9] analyzed the static bending of nanowires, and explained its size-dependent elastic modulus. Using this model, Wang [10] considered the transverse vibration of fluid-conveying nanotube, $\mathrm{Fu}$ et al. [11] studied the nonlinear static and dynamic behaviors of nanobeams, and Assadi and Farshi [12] investigated the size-dependent stability and selfstability of circular nanoplates.

Most of above analyses addressed surface effects on single nanowire. Recently, the double-nanobeam-system (DNBS) has been utilized in nano-optomechanical systems [1318]. The DNBS can be modeled by two one-dimensional nanobeams connected by coupling medium (i.e., van der Waals force, electrostatic force, capillary force, or Casimir force). Frank et al. [14] used electrostatic forces to tune the reconfigurable filters based on two coupled nanobeams model. Karabalin et al. [18] studied the nonlinear dynamics of two elastically coupled nanomechanical resonators and demonstrated that one oscillator could be modified by driving the second oscillator.

In the present paper, we will analyze surface effects on the transverse vibration and axial buckling of DNBS. Our solutions would provide more accurate predictions on the mechanical properties of DNBS and a more reliable 
mechanical model for the design of coupled photonic crystal nanobeams [16].

\section{Surface Effects on Beam Deformation}

The creation of a free surface in a solid leads to excess free energy, which is referred as surface energy, thereby the increase in surface area during deformation will require external work. In addition, the atoms or molecules near a free surface experience a different local environment than that in the interior of the material. As a consequence, the energy density, atom density, and other physical properties in the surface layer will be distinct from those in the bulk.

Surface effects on the mechanical behavior of nanosized elements and nanomaterials can be examined by considering surface energy and/or surface stresses. According to Gibbs [19] and Cammarata [20], the surface stress tensor $\sigma_{\alpha \beta}^{s}$ is related to the surface energy density $\gamma$ through the surface strain tensor $\varepsilon_{\alpha \beta}^{s}$ by

$$
\sigma_{\alpha \beta}^{s}=\gamma \delta_{\alpha \beta}+\frac{\partial \gamma}{\partial \varepsilon_{\alpha \beta}^{s}}
$$

For the deformation of microbeam, only surface stress and surface strain along the longitude direction are of importance, and the one-dimensional and linear form of (1) is

$$
\sigma^{s}=\tau^{0}+E^{s} \mathcal{E}^{s}
$$

where $\tau^{0}=\gamma+\partial \gamma /\left.\partial \varepsilon^{s}\right|_{\varepsilon^{s}=0}$ is the residual surface stress when the bulk is under unstrained and $E^{s}$ is the surface Young's modulus and can be determined either by atomic simulations or experimental measurements $[3,6]$. The ratio of surface energy, surface stress, and surface modulus to the bulk elastic modulus is usually on the order of nanometers. For macroscopic structures, the influence of surface effects can be neglected. However, for nanosized structural elements, the contribution of surface effects becomes quite important and should be accounted for.

According to Laplace-Young equation $[7,9]$, the residual surface stress induces a distributed normal pressure $q(x)$ spanning the beam (as shown in Figure 1(a)), which is given by

$$
q(x)=H \frac{d^{2} w(x)}{d x^{2}}
$$

where $w(x)$ is deflection at the position $x . H$ is a constant related to the residual surface stress and the cross-sectional shape. For a rectangular cross section with width $b$ and height $h$ or a circular cross section with diameter $D$ as shown in Figures 1(b) and 1(c), respectively, one has [7, 9]

$$
H= \begin{cases}2 \tau^{0} b & (\text { rectangular }) \\ 2 \tau^{0} D & (\text { circular })\end{cases}
$$

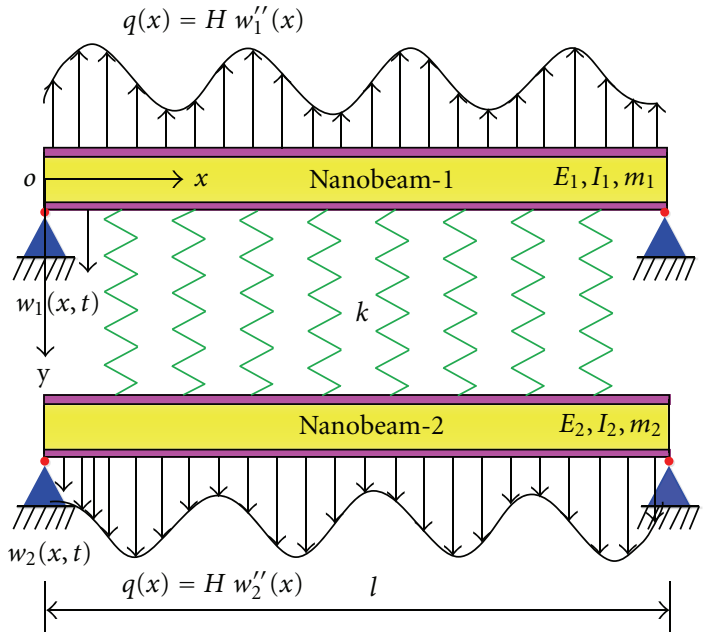

(a)

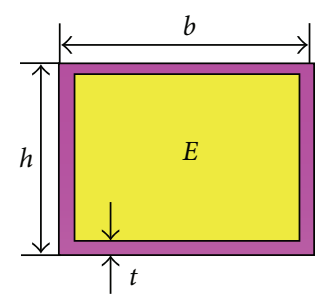

(b)

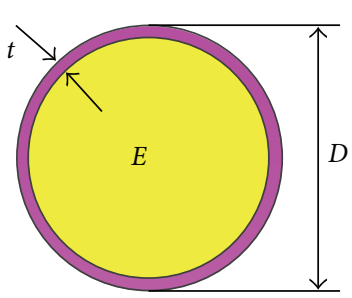

(c)
Figure 1: (a) A double-nanobeam-system, (b) a rectangular cross section with a surface layer, and (c) a circular cross section with a surface layer.

The influence of the second term in (2) can be accounted for by the effective flexural rigidity $(E I)^{*}[7,9]$

$$
(E I)^{*}= \begin{cases}\frac{1}{12} E b h^{3}+\frac{1}{2} E^{s} b h^{2}+\frac{1}{6} E^{s} h^{3} & \text { (rectangular), } \\ \frac{\pi}{64} E D^{4}+\frac{\pi}{8} E^{s} D^{3} & \text { (circular) }\end{cases}
$$

where $E$ is the Young's modulus of the bulk of beam. In what follows, we will consider the vibration and buckling of DNBS by this surface model.

\section{Vibration of Double-Nanobeam-System}

Consider a double-nanobeam-system as illustrated in Figure 1(a). Two nanobeams with identical length $l$ are connected by distributed springs with stiffness $k$. In physical nature, the springs could represent the effects of electrostatic force, nano-optomechanical-induced force, van der Waals force, or elastic medium, which can be adjusted by the electrical potential difference or the distance between two nanobeams [14]. Denote the elastic modulus, mass density, cross-section area, and second moment of inertia of the $i$ th beam by $E_{i}, \rho_{i}, A_{i}$, and $I_{i}(i=1,2)$, respectively. Since the DNBS usually has a large length/depth ratio $(l / D \gg 10)[13]$, it is reasonable to neglect the effect of shear deformation and 
rotary inertia, and adopt the Euler-Bernoulli beam theory to predict its mechanical behaviors.

Denote the deflections of two nanobeams by $w_{1}(x, t)$ and $w_{2}(x, t)$, respectively. Account for surface effects in Section 2, the differential equations of the free vibration of DNBS can be obtained as

for nano-beam-1,

$$
\left(E_{1} I_{1}\right) * \frac{\partial^{4} w_{1}}{\partial x^{4}}-H \frac{\partial^{2} w_{1}}{\partial x^{2}}+k\left(w_{1}-w_{2}\right)+\rho_{1} A_{1} \frac{\partial^{2} w_{1}}{\partial t^{2}}=0,
$$

and for nano-beam-2,

$$
\left(E_{2} I_{2}\right) * \frac{\partial^{4} w_{2}}{\partial x^{4}}-H \frac{\partial^{2} w_{2}}{\partial x^{2}}+k\left(w_{2}-w_{1}\right)+\rho_{2} A_{2} \frac{\partial^{2} w_{2}}{\partial t^{2}}=0 .
$$

In practical applications, the two nanobeams in DNBS are usually identical; therefore, in present paper we assume

$$
\begin{gathered}
E_{1} I_{1}=E_{2} I_{2}=E I, \quad\left(E_{1} I_{1}\right)^{*}=\left(E_{2} I_{2}\right)^{*}=(E I)^{*}, \\
\rho_{1} A_{1}=\rho_{2} A_{2}=m .
\end{gathered}
$$

It should be noted that more general cases can also be achieved but in a more complicated form [21].

For convenience in analysis, we introduce the relative movement of two beams as [22]

$$
w(x, t)=w_{1}(x, t)-w_{2}(x, t) .
$$

Then

$$
w_{1}(x, t)=w(x, t)+w_{2}(x, t) .
$$

Subtracting (6) from (7) gives

$$
\begin{gathered}
(E I)^{*} \frac{\partial^{4} w}{\partial x^{4}}-H \frac{\partial^{2} w}{\partial x^{2}}+2 k w+m \frac{\partial^{2} w}{\partial t^{2}}=0, \\
(E I)^{*} \frac{\partial^{4} w_{2}}{\partial x^{4}}-H \frac{\partial^{2} w_{2}}{\partial x^{2}}+m \frac{\partial^{2} w_{2}}{\partial t^{2}}=k w .
\end{gathered}
$$

When surface effects are ignored $\left(H=E^{s}=0\right)$ and single beam $(k=0)$ is considered, (12) reverts to the vibration equations of a single Euler beam.

In order to display the surface effects, we consider a simple case, in which both beams are simply supported at their ends. The boundary conditions are given by

$$
\begin{gathered}
w_{1}(0, t)=w_{2}(0, t)=0, \quad w_{1}(l, t)=w_{2}(l, t)=0, \\
\frac{\partial^{2} w_{1}(0, t)}{\partial x^{2}}=\frac{\partial^{2} w_{2}(0, t)}{\partial x^{2}}=0, \\
\frac{\partial^{2} w_{1}(l, t)}{\partial x^{2}}=\frac{\partial^{2} w_{2}(l, t)}{\partial x^{2}}=0 .
\end{gathered}
$$

Thus, the boundary conditions associated with (11) become

$$
\begin{array}{rlrl}
w(0, t) & =0, & & w(l, t)=0, \\
\frac{\partial^{2} w(0, t)}{\partial x^{2}} & =0, & \frac{\partial^{2} w(l, t)}{\partial x^{2}}=0 .
\end{array}
$$

Assuming that the relative motion $w(x, t)$ is one of its natural modes of vibration of DNBS, the boundary condition (16) can be satisfied by the following vibration displacement:

$$
\begin{array}{r}
w^{(n)}(x, t)=\sin \left(\frac{n \pi x}{l}\right)\left[C \cos \left(p_{n} t\right)+D \sin \left(p_{n} t\right)\right], \\
n=1,2,3 \ldots,
\end{array}
$$

where $p_{n}$ is the natural frequency of $n$th mode.

To discuss the vibration and buckling of DNBS, three typical cases including out-of-phase sequence, in-phase sequence, and one-beam being stationary as shown in Figures 2(a), 2(b), and 2(c), are considered, respectively.

3.1. Out-of-Phase Vibration. In this case, both nanobeams vibrate out-of-phase, and $w_{1}(x, t)-w_{2}(x, t) \neq 0$, as shown in Figure 2(a). Substituting (17) into (11), one can obtain the natural frequency of DNBS in the out-of-phase vibration as

$$
p_{n}=\sqrt{\frac{n^{4} \pi^{4}}{l^{4}} \frac{(E I)^{*}}{m}+\frac{n^{2} \pi^{2}}{l^{2}} \frac{H}{m}+\frac{2 k}{m}}, \quad n=1,2,3, \ldots
$$

When surface effects are neglected $\left(H=E^{s}=0\right)$, the natural frequency reduces to the classical double Euler beam solution $P_{n}^{0}[22]$,

$$
p_{n}^{0}=\sqrt{\frac{n^{4} \pi^{4}}{l^{4}} \frac{E I}{m}+\frac{2 k}{m}}, \quad n=1,2,3, \ldots
$$

3.2. In-Phase Vibration. In the case of in-phase vibration as shown in Figure 2(b), two nanobeams vibrate synchronously, thus the relative displacement between them disappears $\left(w_{1}(x, t)-w_{2}(x, t)=0\right)$. Therefore, any one of the two beams could represent the vibration of the coupled vibration system. Following a similar procedure as that in out-of-phase vibration, one can determine the frequency as

$$
p_{n}=\sqrt{\frac{n^{4} \pi^{4}}{l^{4}} \frac{(E I)^{*}}{m}+\frac{n^{2} \pi^{2}}{l^{2}} \frac{H}{m}}, \quad n=1,2,3, \ldots
$$

It is seen that the interaction between nanobeams does not affect the natural frequency of DNBS for in-phase vibration, since two beams vibrate synchronously. For this vibrating mode, the vibration frequency is just as the same as that of the single Euler beam with surface effects [7].

3.3. One Nanobeam Being Stationary. Another vibrating mode of interest is one nanobeam being stationary (i.e., $\left.w_{2}(x, t)=0\right)$, as shown in Figure 2(c). In this case, the vibration equation (11) reduces to

$$
(E I)^{*} \frac{\partial^{4} w}{\partial x^{4}}-H \frac{\partial^{2} w}{\partial x^{2}}+k w+m \frac{\partial^{2} w}{\partial t^{2}}=0 .
$$

In this context, the DNBS behaves as if nanobeam-1 is supported on an elastic medium. Similarly, one obtains the natural frequency of beam as

$$
p_{n}=\sqrt{\frac{n^{4} \pi^{4}}{l^{4}} \frac{(E I)^{*}}{m}+\frac{n^{2} \pi^{2}}{l^{2}} \frac{H}{m}+\frac{k}{m}}, \quad n=1,2,3, \ldots
$$




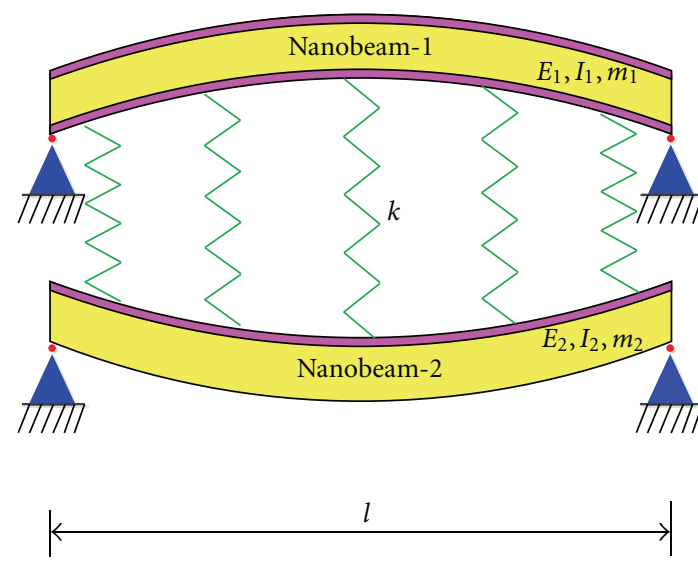

(a)
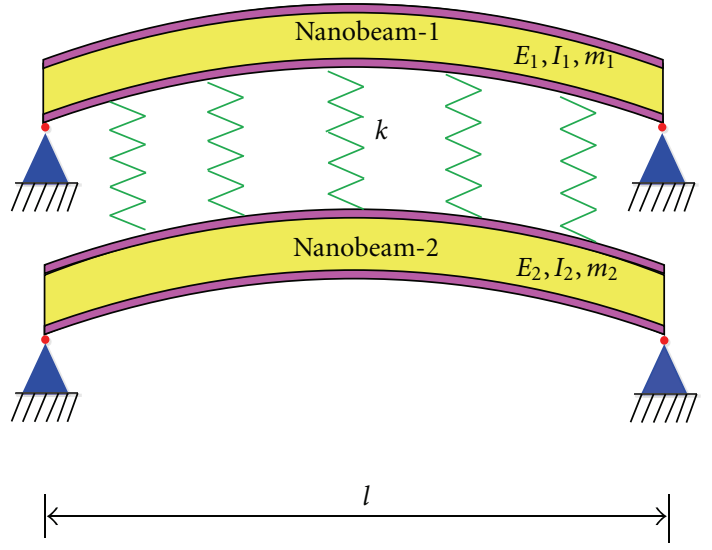

(b)

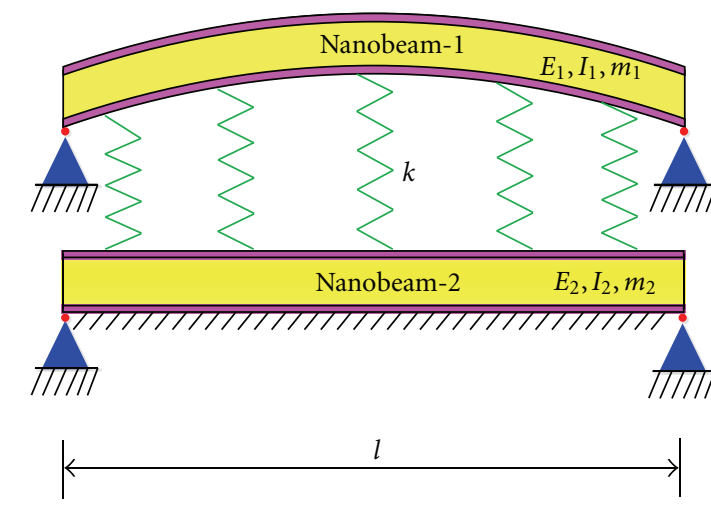

(c)

FIGURE 2: Three different deformation modes of DNBS: (a) out-of-phase deformation, (b) in-phase deformation, and (c) one beam being stationary.

Comparing (18), (20), and (22), it is noticed that surface effects have the same contribution to these three vibration modes, but the influence of beam interaction is distinct in different vibration modes. The interaction between beams tends to increase the natural frequency for vibration modes other than in-phase vibration.

3.4. Example and Discussion. To illustrate surface effects on the vibration of DNBS quantitatively, we consider a DNBS consisting of two silver nanowires with circular cross section. The material constants of nanowires are $E=76 \mathrm{GPa}, \nu=0.3$, and the surface properties $\tau^{0}=0.89 \mathrm{~N} / \mathrm{m}$ and $E^{s}=1.22 \mathrm{~N} / \mathrm{m}$ [9]. To examine the influence of beam interaction, the spring stiffness has been taken from $2 \times 10^{4} \mathrm{~N} / \mathrm{m}^{2}$ to $2 \times 10^{7} \mathrm{~N} / \mathrm{m}^{2}$ [23]. We also take a length/diameter aspect ratio as $l / D=20$ in the following analysis.

Since surface effects are just the same in three vibration modes, here we consider surface effects and beam interaction on only the out-phase vibration. Figure 3 displays the variation of normalized first-order natural frequency $P_{1} / P_{1}^{0}$ with respect to the wire diameter. It is seen that when the diameter reduces to nanometers, the vibration frequency depends on the absolute size of nanobeam, which is clearly distinct from the prediction of conventional elasticity. As the diameter decreases, the contribution of surface effects gets important and tends to increase the natural frequency. It is also noticed that surface effects are more prominent for a small spring constant corresponding to a weak beam interaction. With the spring constant increasing, the influence of surface effects becomes relatively unimportant compared to the beam interaction. The higher-order natural frequency of DNBS is also plotted in Figure 4, in which the spring stiffness $k$ is taken as $2 \times 10^{4} \mathrm{~N} / \mathrm{m}^{2}$. It is found that surface effects are more significant for low-order natural frequency and declines dramatically for high-order frequency.

\section{Axial Buckling of Double-Nanobeam-System}

It is also of interest to consider the axial buckling of DNBS. Accounting for surface effects stated in Section 2, the buckling equations of two nanobeams subjected to axial compressive forces $N_{1}$ and $N_{2}$ can be expressed as

for beam one,

$$
\left(E_{1} I_{1}\right) \frac{\partial^{4} w_{1}}{\partial x^{4}}+\left(N_{1}-H\right) \frac{\partial^{2} w_{1}}{\partial x^{2}}+k\left(w_{1}-w_{2}\right)=0
$$




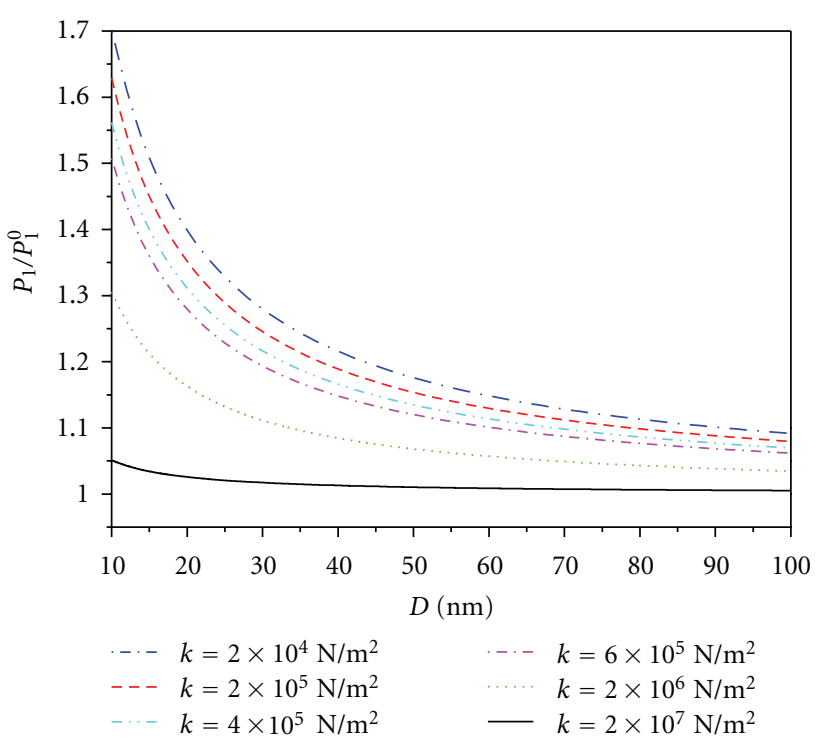

FIGURE 3: Normalized first-order natural frequency of DNBS versus the wire diameter.

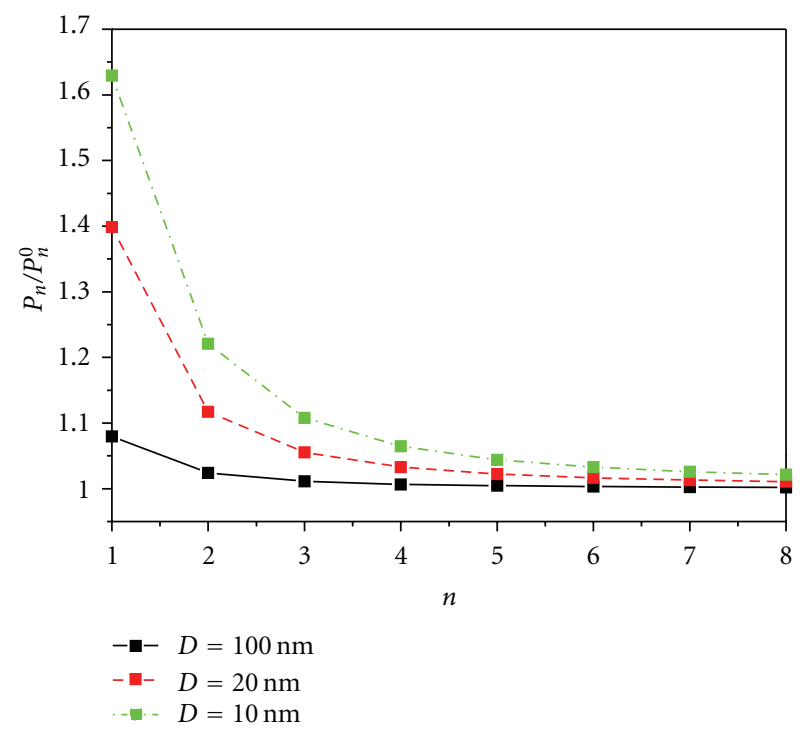

FIGURE 4: Normalized natural frequency of different modes for outof-phase vibration.

and for beam two,

$$
\left(E_{2} I_{2}\right) * \frac{\partial^{4} w_{2}}{\partial x^{4}}+\left(N_{2}-H\right) \frac{\partial^{2} w_{2}}{\partial x^{2}}+k\left(w_{2}-w_{1}\right)=0 .
$$

Assume the two beams are identical for simplification and $N_{1}=N_{2}$, we get

$$
\begin{gathered}
(E I) * \frac{\partial^{4} w}{\partial x^{4}}+(N-H) \frac{\partial^{2} w}{\partial x^{2}}+2 k w=0, \\
(E I)^{*} \frac{\partial^{4} w_{2}}{\partial x^{4}}+(N-H) \frac{\partial^{2} w_{2}}{\partial x^{2}}=k w .
\end{gathered}
$$

Similar to those of vibration modes, the buckling of DNBS can also be categorized into the out-of-phase buckling, in-phase buckling, and buckling with one beam being stationary. We also consider only the boundary conditions for ends of two beams being simply supported, as described by (16). The following buckling mode satisfies the boundary condition

$$
w=W \sin \left(\frac{n \pi x}{l}\right) .
$$

For out-of-phase buckling, substitution of (27) into (25) yields

$$
(E I)^{*}\left(\frac{n \pi}{l}\right)^{4}+(H-N)\left(\frac{n \pi}{l}\right)^{2}+2 k=0 .
$$

Consequently, the critical buckling load is derived as

$$
N_{\mathrm{cr}}=\frac{n^{2} \pi^{2}(E I)^{*}}{l^{2}}+\frac{2 k l^{2}}{n^{2} \pi^{2}}+H, \quad n=1,2,3, \ldots
$$

For the case without surface effects $\left(H=E^{s}=0\right)$, the critical load reduces to the classical solution

$$
N_{\mathrm{cr}}^{0}=\frac{n^{2} \pi^{2} E I}{l^{2}}+\frac{2 k l^{2}}{n^{2} \pi^{2}}, \quad n=1,2,3, \ldots
$$

Similarly, the critical load of buckling for in-phase buckling can be readily given as

$$
N_{\mathrm{cr}}=\frac{n^{2} \pi^{2}(E I)^{*}}{l^{2}}+H, \quad n=1,2,3, \ldots
$$

This coincides with the solution of axial buckling of a single nanowire [8].

Also, for the case of buckling with one beam being stationary, the critical load is expressed as

$$
N_{\mathrm{cr}}=\frac{n^{2} \pi^{2}(E I)^{*}}{l^{2}}+\frac{k l^{2}}{n^{2} \pi^{2}}+H, \quad n=1,2,3, \ldots
$$

For the axial buckling of DNBS, it is found again that the influence of surface effects is just the same in three buckling modes. For in-phase buckling, the beam interaction has no influence on the critical load, while for other buckling modes, the beam interaction will enhance the critical load of DNBS.

To demonstrate surface effects on the buckling of NDBS, we consider two circular silver nanowires with $E=76 \mathrm{GPa}$, $v=0.3$, and surface properties $\tau^{0}=0.89 \mathrm{Nm}$ and $E^{s}=$ $1.22 \mathrm{Nm}$ [9]. The interaction between them is modeled by the coupling stiffness $k$ varying from $2 \times 10^{4} \mathrm{~N} / \mathrm{m}^{2}$ to $2 \times$ $10^{7} \mathrm{~N} / \mathrm{m}^{2}$ [23]. For out-of-phase buckling, Figure 5 displays the critical compressive force $N_{\mathrm{cr}} / N_{\mathrm{cr}}^{0}$ with respect to the wire diameter. The normalized critical force of buckling exhibits a distinct dependence on the characteristic size of the nanowires, which is different from the conventional elasticity. The influence of surface effects become significant as the diameter decreases in the range of nanometers and usually raises the critical buckling load of DNBS. Moreover, surface effects are more prominent for weak beam interaction than for stiff interaction. 


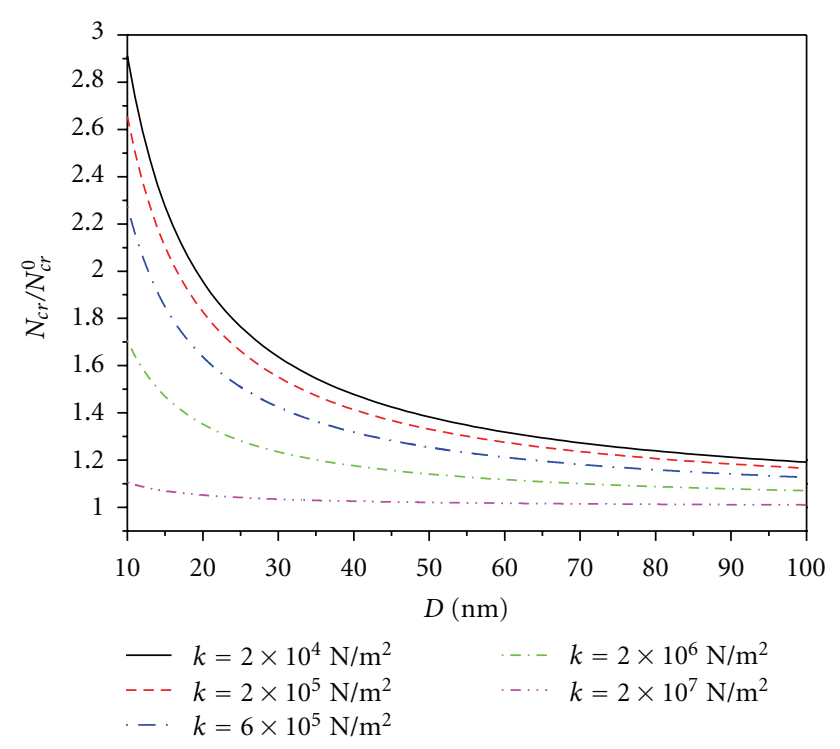

Figure 5: Normalized critical load of DNBS versus the nanowire diameter.

\section{Conclusions}

Based on a modified Euler-Bernoulli beam theory, we have analyzed surface effects on the transverse vibration and axial buckling of DNBS. The natural frequency and critical compression force are obtained analytically. The results show that both surface elasticity and residual surface tension affect the natural frequency and buckling load of DNBS when the cross section of nanowires shrinks to nanometers. Surface effects play the same impact in three deformation modes no matter for vibration or axial buckling and evidently enhance the natural frequency and critical load. In contrast, the influence of beam interaction is clearly distinct in different deformation modes. The present study might be helpful for the design of double-nano-beam-based nano-optomechanical systems and nanoelectromechanical systems.

\section{Acknowledgments}

The supports from the National Natural Science Foundation (Grant no. 11072186) and the NCET program and SRF for ROCS of MOE are acknowledged.

\section{References}

[1] Y. Cui, Q. Wei, H. Park, and C. M. Lieber, "Nanowire nanosensors for highly sensitive and selective detection of biological and chemical species," Science, vol. 293, no. 5533, pp. 1289-1292, 2001.

[2] M. E. Gurtin, J. Weissmüller, and F. Larché, "A general theory of curved deformable interfaces in solids at equilibrium," Philosophical Magazine A, vol. 78, no. 5, pp. 1093-1109, 1998.

[3] R. E. Miller and V. B. Shenoy, "Size-dependent elastic properties of nanosized structural elements," Nanotechnology, vol. 11, no. 3, pp. 139-147, 2000.
[4] C. Q. Ru, "Simple geometrical explanation of GurtinMurdoch model of surface elasticity with clarification of its related versions," Science China, vol. 53, no. 3, pp. 536-544, 2010.

[5] C. Q. Chen, Y. Shi, Y. S. Zhang, J. Zhu, and Y. J. Yan, "Size dependence of Young's modulus in $\mathrm{ZnO}$ nanowires," Physical Review Letters, vol. 96, no. 7, Article ID 075505, pp. 1-4, 2006.

[6] S. Cuenot, C. Frétigny, S. Demoustier-Champagne, and B. Nysten, "Surface tension effect on the mechanical properties of nanomaterials measured by atomic force microscopy," Physical Review B, vol. 69, no. 16, Article ID 165410, 5 pages, 2004.

[7] G.-F. Wang and X.-Q. Feng, "Effects of surface elasticity and residual surface tension on the natural frequency of microbeams," Applied Physics Letters, vol. 90, no. 23, Article ID 231904, 2007.

[8] G.-F. Wang and X.-Q. Feng, "Surface effects on buckling of nanowires under uniaxial compression," Applied Physics Letters, vol. 94, no. 14, Article ID 141913, 2009.

[9] J. He and C. M. Lilley, "Surface effect on the elastic behavior of static bending nanowires," Nano Letters, vol. 8, no. 7, pp. 1798-1802, 2008.

[10] L. Wang, "Vibration analysis of fluid-conveying nanotubes with consideration of surface effects," Physica E, vol. 43, no. 1, pp. 437-439, 2010.

[11] Y. Fu, J. Zhang, and Y. Jiang, "Influences of the surface energies on the nonlinear static and dynamic behaviors of nanobeams," Physica E, vol. 42, no. 9, pp. 2268-2273, 2010.

[12] A. Assadi and B. Farshi, "Size dependent stability analysis of circular ultrathin films in elastic medium with consideration of surface energies," Physica E, vol. 43, no. 5, pp. 1111-1117, 2011.

[13] M. Eichenfield, R. Camacho, J. Chan, K. J. Vahala, and O. Painter, "A picogram- and nanometre-scale photonic-crystal optomechanical cavity," Nature, vol. 459, no. 7246, pp. 550$555,2009$.

[14] I. W. Frank, P. B. Deotare, M. W. McCutcheon, and M. Lonèar, "Programmable photonic crystal nanobeam cavities," Optics Express, vol. 18, no. 8, pp. 8705-8712, 2010.

[15] M. W. McCutcheon, P. B. Deotare, Y. Zhang, and M. Lončar, "High- Q transverse-electric/transverse-magnetic photonic crystal nanobeam cavities," Applied Physics Letters, vol. 98, no. 11, Article ID 111117, 3 pages, 2011.

[16] P. B. Deotare, M. W. McCutcheon, I. W. Frank, M. Khan, and M. Lončar, "Coupled photonic crystal nanobeam cavities," Applied Physics Letters, vol. 95, no. 3, Article ID 031102, 3 pages, 2009.

[17] Q. Lin, J. Rosenberg, D. Chang et al., "Coherent mixing of mechanical excitations in nano-optomechanical structures," Nature Photonics, vol. 4, no. 4, pp. 236-242, 2010.

[18] R. B. Karabalin, M. C. Cross, and M. L. Roukes, "Nonlinear dynamics and chaos in two coupled nanomechanical resonators," Physical Review B, vol. 79, no. 16, Article ID 165309, 5 pages, 2009.

[19] J. W. Gibbs, The Scientific Papers of J. Willard Gibbs. Vol 1: Thermodynamics, Longmans and Green, New York, NY, USA, 1993.

[20] R. C. Cammarata, "Surface and interface stress effects in thin films," Progress in Surface Science, vol. 46, no. 1, pp. 1-38, 1994.

[21] S. G. Kelly and S. Srinivas, "Free vibrations of elastically connected stretched beams," Journal of Sound and Vibration, vol. 326, no. 3-5, pp. 883-893, 2009.

[22] H. V. Vu, A. M. Ordonez, and B. H. Karnopp, "Vibration of a double-beam system," Journal of Sound and Vibration, vol. 229, no. 4, pp. 807-822, 2000. 
[23] J. Zhu, C. Q. Ru, and A. Mioduchowski, "Instability of a large coupled microbeam array initialized at its two ends," Journal of Adhesion, vol. 83, no. 3, pp. 195-221, 2007. 

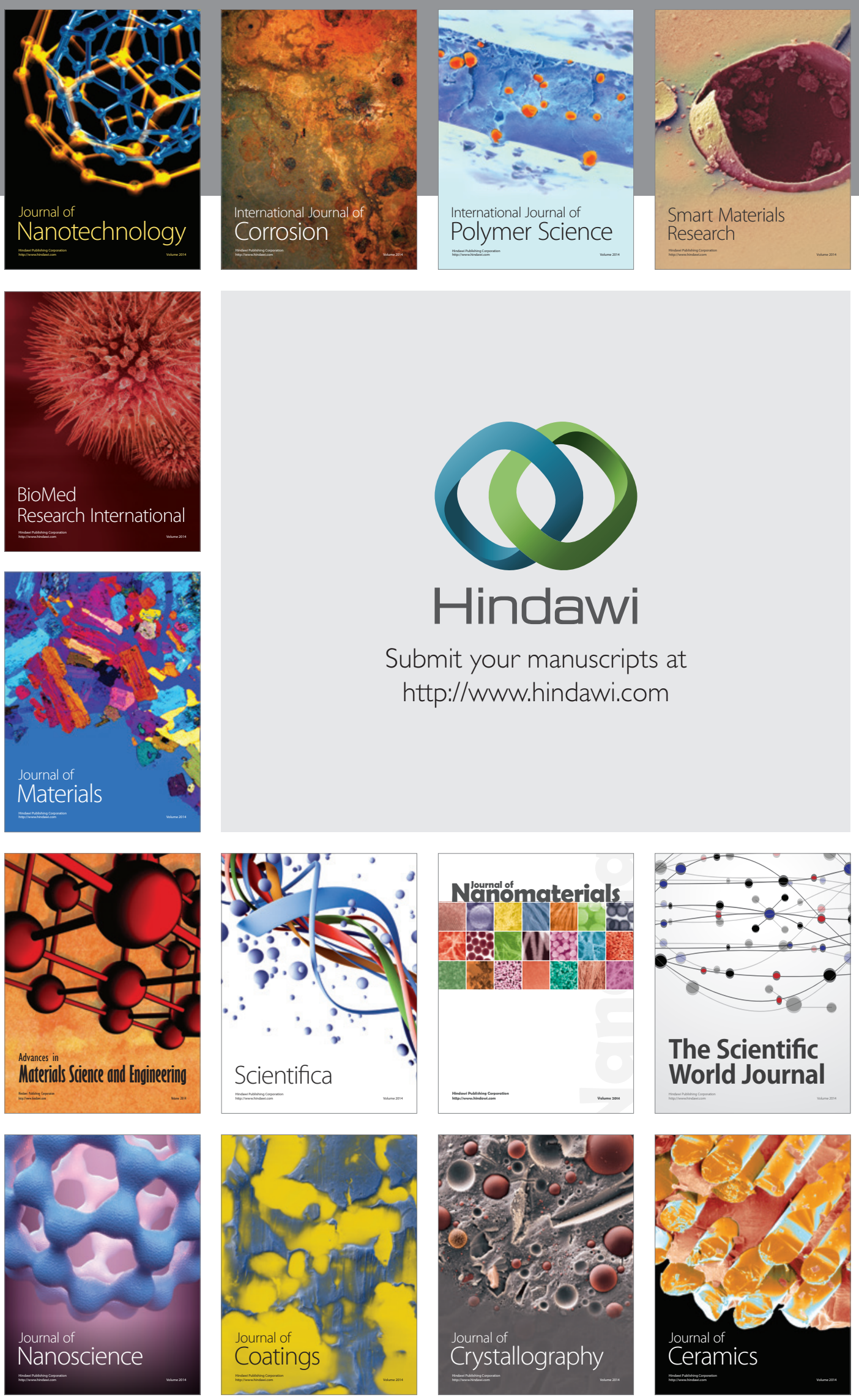

The Scientific World Journal

Submit your manuscripts at

http://www.hindawi.com

\section{World Journal}

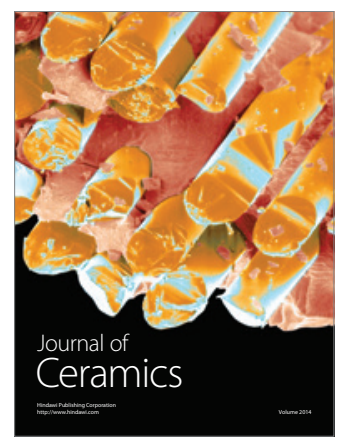

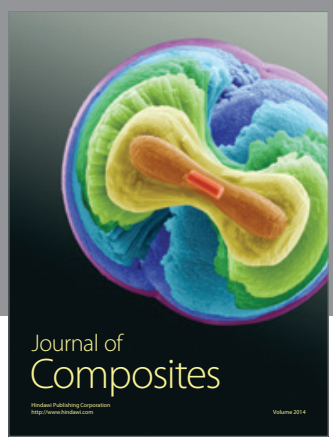
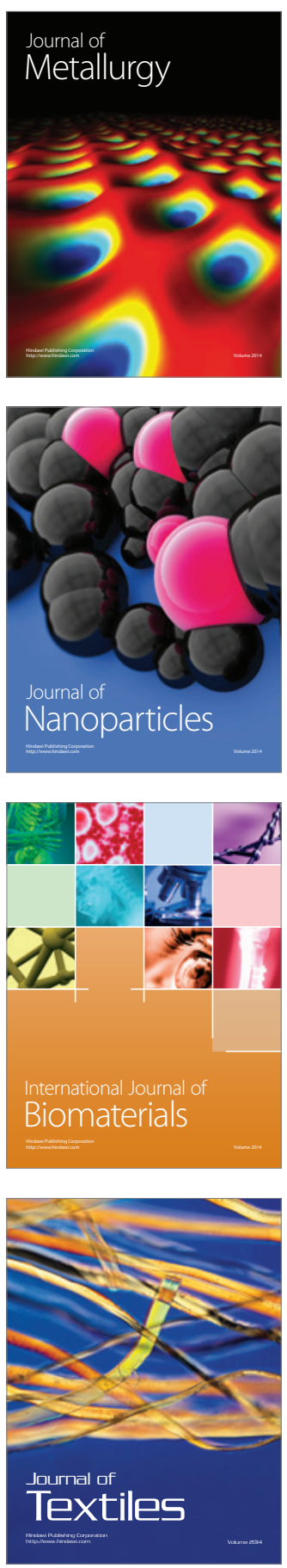